\title{
Clinical and economic burden of idiopathic pulmonary fibrosis in Quebec, Canada
}

This article was published in the following Dove Press journal:

ClinicoEconomics and Outcomes Research

\author{
Jean-Eric Tarride ${ }^{1,2}$ \\ Robert B Hopkins ${ }^{1,2}$ \\ Natasha Burke ${ }^{1,2}$ \\ Jason R Guertin 3,4 \\ Daria O'Reilly,2 \\ Charlene D Fell ${ }^{5}$ \\ Genevieve Dion ${ }^{6}$ \\ Martin Kolb ${ }^{7}$
}

'Department of Health Research Methods, Evidence and Impact, Faculty of Health Sciences, McMaster University, Hamilton, ON, Canada; 2Programs for Assessment of Technology in Health (PATH), The Research Institute of St. Joe's Hamilton, St. Joseph's Healthcare Hamilton, Hamilton, ON, Canada; ${ }^{3}$ Department of Social and Preventive Medicine, Laval University, Quebec City, QC, Canada; ${ }^{4}$ Centre de recherche du CHU de Québec Université Laval, Axe Santé des Populations et Pratiques Optimales en Santé, Hôpital du St-Sacrement, Quebec City, QC, Canada; ${ }^{5}$ Division of Respirology, Department of Medicine, University of Calgary, Calgary, AB, Canada; ' $Q$ Quebec Heart and Lung Institute, Laval University, Quebec City, QC, Canada; ${ }^{7}$ Division of Respirology, Department of Medicine, Faculty of Health Sciences, McMaster University, Hamilton, ON, Canada

Correspondence: Jean-Eric Tarride Department of Health Research Methods, Evidence and Impact, Faculty of Health Sciences, McMaster University, 43 Charlton Ave E, 2nd Floor, Hamilton, ON, L8N IY3, Canada

Tel + I 905522 II 55 ext 3702 I

$\mathrm{Fax}+\mathrm{I} 9025220568$

Email tarride@mcmaster.ca
Background: Idiopathic pulmonary fibrosis (IPF), although rare, is a severe and costly disease. Objective: To estimate the clinical and economic burden of IPF over multiple years before and after diagnosis using comprehensive administrative databases for the province of Quebec, Canada. Methods: Several administrative databases from Quebec, providing information on hospital care, community care, and pharmaceuticals, were linked over a 5-year period ending March 31, 2011, which was before approval of antifibrotic drugs in Canada. Prevalent and incident IPF cases were defined using International Classification Disease-10-CA codes and International Classification Disease-9-CM codes. We used a broad definition that excluded cases with subsequent diagnosis of other interstitial lung diseases and a narrow definition that required further diagnostic testing to confirm IPF diagnosis. Incident cases had an IPF code in a particular year without any IPF code in the 2 previous years. Health care resource utilization before and after the index diagnosis date was determined and costs calculated. Costs were expressed in 2016 Canadian dollars.

Results: Over 5-years, 10,579 (mean age: 76.4; 58\% male) satisfied the broad definition of IPF and 8,683 (mean age: $74.5 ; 57 \%$ male) satisfied the narrow definition ( $82 \%$ of broad). Incidences of IPF overall were 25.8 and $21.7 / 100,000$ population for broad and narrow definitions, respectively. Three-year survival was $40 \%$ and $37 \%$ in broad and narrow cohorts, respectively. For both cohorts, health care resource utilization and costs increased several years before diagnosis $(\$ 2,721$ and $\$ 7,049$ /patient 5 years and 2 years prior to diagnosis using a broad definition, respectively) and remained elevated for multiple years post diagnosis $(\$ 12,978$ and $\$ 8,267$ at 2 and 3 years postdiagnosis).

Conclusion: Health care resource utilization and costs of IPF increase many years prior to diagnosis. Incorporating multiyear annual costs before and after diagnosis results in a higher estimate of the economic burden of IPF than previous studies using a 1-year time frame.

Keywords: cost of illness, health care utilization, incidence, idiopathic pulmonary fibrosis, Canada

\section{Plain language summary}

Idiopathic pulmonary fibrosis (IPF), although rare, is a severe and costly disease. Using comprehensive health administrative data from the province of Quebec, Canada (population 8.0 million), we estimated the amount of health care resources used in the years leading up to the index IPF diagnosis and for up to 3 years following the diagnosis. Costs to the health care system were calculated. Annual health care costs for patients with IPF begin to increase prior to diagnosis, which is consistent with the natural history of the disease. With the progression of the disease, a patient experiences declines in health status, which leads to diagnostic investigations to establish the diagnosis. Five years prior to diagnosis, 
the average yearly cost was $\$ 2,721$ per patient. At 2 years prior to diagnosis, the average yearly cost rose to $\$ 7,049$ per patient. In the year following the diagnosis of IPF, average health care costs were $\$ 12,978$ per patient, and remained elevated for multiple years. Three-year survival was $37 \%-40 \%$ depending on the definition used to identify IPF cases. This study showed that the economic burden of IPF was higher than previous studies that used a 1-year time frame.

\section{Introduction}

Idiopathic pulmonary fibrosis (IPF) is a chronic progressive fibrotic disease of the lung. ${ }^{1}$ The typical age of onset is $>60$ years, and symptoms and signs typically develop over 6 months to several years and include dyspnea on exertion and nonproductive cough. The progression of IPF is hallmarked by rapidly declining lung function until lung transplant or mortality. The prevalence of IPF appears to be increasing, not only because of an aging population, but also due to an increased awareness of IPF among patients and clinicians, and an improved ability to diagnose the disease confidently by applying advances in computed tomography (CT) imaging. ${ }^{2}$ In Canada, we recently estimated the incidence of IPF at $18.7 / 100,000 .^{3}$

IPF is a progressive disease with a survival rate as little as $50 \%$ after 3 years and has significant impairment on healthrelated quality of life (HRQoL). ${ }^{3}$ It has been shown that the HRQoL associated with IPF is lower than HRQoL associated with COPD Global Initiative on Obstructive Lung Disease (GOLD) stage IV, which is partially explained by the high number of comorbidities associated with IPF (eg, pulmonary hypertension, gastroesophageal reflux disease, obesity, lung cancer, and emphysema). ${ }^{2,4}$ In addition to the clinical and human burden, IPF is a costly disease, as reported in a few recent US studies. For example, the annual health care costs per IPF patient increased from \$USD10,000/yr in the year before the diagnosis of IPF to \$USD20,000 in the year after the diagnosis. ${ }^{5}$ Another US study has also documented an increase in health care utilization in the year following the index diagnosis date. ${ }^{6}$ However, it is currently unknown if the costs in the multiple years before diagnosis show any pattern, or more importantly, if the elevated costs that occur in the year postdiagnosis persist over the years throughout disease progression.

To better inform decision-makers, physicians, and patients, the objective of this study was to estimate mortality and health care resource utilization and costs that occur over multiple years pre- and postdiagnosis for patients newly diagnosed with IPF using a very comprehensive public reporting system in the province of Quebec in Canada.

\section{Methods}

The clinical and economic burden of IPF was estimated using 5 years of administrative data from the province of Quebec (population 8.0 million, 23\% of Canada). Data were used to identify unique cases of IPF and estimate the health care resource utilization and costs before and after the IPF index diagnosis date. When necessary, data from our previous study based on Canadian non-Quebec data ${ }^{3}$ were used to fill gaps in the Quebec data. The research ethics board of the Quebec Heart and Lung Institute approved the study and access to the data was authorized by the Commission d'accès à l'information.

\section{Data sources}

The focus of the analysis is the province of Quebec where all citizens are eligible for comprehensive public health care. Deidentified record-level linkable administrative data with International Classification Disease (ICD)-10-CA and ICD-9-CM diagnosis codes were obtained from the Régie de l'Assurance Maladie du Québec (RAMQ) for the 5 fiscal years April 1, 2006 to March 31, 2011. Data linkage included information on vital statistics (date of birth, first record in databases, date of death), hospital admissions (including ICD-10-CA diagnostic codes and interventions), medical services (ICD-9-CM diagnosis codes, billings count and fees charged, specialist or general practice, and location of service), and prescribed medications covered by RAMQ (drug identification number, American Hospital Formulary Service class, cost).

\section{Case definition}

IPF cases were identified as having at least one physician service with a billing code of 516.3 (idiopathic fibrosing alveolitis) or having a hospital admission with a primary or secondary ICD-10-CA code of J84.1 (idiopathic pulmonary fibrosis) during the study period. Incident cases were defined as having an IPF code in a particular year without any IPF code in the 2 previous years. Due to our definition of incident cases and data availability (2006-2011), the minimum and maximum years of data before IPF diagnosis is 2 (2008 incident cases) and 5 years (2011 incident cases), respectively. The maximum years of data postdiagnosis was 3 (diagnosed in 2008).

Similar to other work, ${ }^{3,5}$ a broad definition IPF case was defined as having either the ICD-9 or ICD-10 code of IPF for hospital admissions or physician billings databases over the 5 years without having a subsequent code for over 200 other interstitial diseases (including connective tissue diseases, sclerosis, sarcoidosis, etc). A narrow definition was defined as having a broad definition plus an IPF diagnosis 
recorded after a CT scan of the thorax, surgical lung biopsy, or bronchoscopy.

\section{Morbidity and mortality}

Aligned with previous work, ${ }^{3}$ comorbid conditions associated with IPF were identified in the physicians' database through ICD-9-CM codes. Kaplan-Meier survival estimates were used to estimate survival of an incident cohort of IPF patients identified in 2008 allowing for 3 years of follow-up.

\section{Health care resource utilization and costs}

For each case, health care resource utilization and costs were determined before and after the index diagnosis date in terms of hospitalizations, physician billings, emergency room (ER) visits, prescribed medications, and home care and long-term care.

\section{Hospital admissions and costs}

In addition to length of stay, Quebec hospitalization data captured in the Maintenance et Exploitation de Données pour l'Étude de la Clientèle Hospitalière (MedEcho) database include ICD-10 diagnosis codes (for any admitting code, main problem, secondary problem, or cause of death) and intervention codes. However, the MedEcho database does not provide information on the costs associated with each hospitalization. To overcome this limitation, information on hospitalization data from all Canadian provinces except Quebec based on our previous study were used to assign a resource intensity weight (RIW) to IPF hospitalizations in Quebec. RIW (a relative resource number assigned to each hospitalization where a RIW of 1.0 equals an average cost of stay) is a standard Canadian costing methodology developed by the Canadian Institute of Health Information to determine the cost of hospitalization in Canada. ${ }^{7}$ Each admission has a separate RIW adjusted by case mix group, age, comorbidity levels, flagged interventions, number of intervention events, out-of-hospital interventions, and type of hospital. Since the unit costs of an RIW are available for Canada overall and for each province, ${ }^{8}$ the RIW unit for Quebec for 2016 (\$5,795 per RIW unit) was used to calculate the cost associated with IPF hospitalizations in Quebec. The RIW captured the hospital budget portion of an admission, while the physician billings that occurred as part of the admission were captured in the physician database.

\section{Physician services and costs}

Information on physician services was derived from the Quebec physicians' billing database (Rémunération à l'acteMédecine or RAMQ) which provides the dollar value of each medical service performed, such as consultations, request for laboratory for diagnostic tests, or referral fees, at all locations where care is provided (ie, hospitals, ER, or physicians' offices). We limited the count of the number of physicians to one per day, although a patient may have been seen more than once per day or by more than one physician per day. Although the specific type of physician was not available (eg, respirologist), an indication of general practice or specialty was available and used for the analyses.

\section{ER visits and costs}

There is no unique record for ER visits, and instead we relied on the RAMQ database which provides the location where a medical act was performed by a physician as it is mandatory to report an ER code $(0 \times 7)$ for any service provided in the ER in Quebec. Since a description of the health care resource utilization or an estimate of the cost associated with an ER visit was not provided in the RAMQ database, we relied on unpublished information from our previous IPF study ${ }^{3}$ to derive the average cost of an ER visit in Quebec at CAD \$376. Note that this cost does not include the physician billings.

\section{Prescribed medication use and costs}

For each prescription filled, the Services Médicaments database provides the drug identification number ( 8 digit number assigned by Health Canada to a drug product prior to being marketed in Canada) and the total cost of prescriptions filled for RAMQ beneficiaries (eg, residents of Quebec 65 years of age or older, anyone not eligible for a private plan, recipients of last-resort financial assistance, and children of persons covered by the public plan).

\section{Home care and long-term care use and costs}

The MedEcho hospital database provides a record of residency type before and after each admission which was used to determine the number of IPF patients who were discharged each year with home care services or long-term care residency. In the absence of a Quebec-based home care database, we used the cost of home care from our national publication ${ }^{3}$ and assumed that the mean duration of home care in Quebec was 194 days (with a maximum of 365 days) and, using an average weekly cost of $\$ 306$, the average cost of home care was $\$ 8,390$. The weekly cost of home care was based on the relative resource intensity Case Mix Index of 1.73, where a Case Mix Index of 1 is equivalent to the average cost per week for all home care in Ontario (\$177.07). Similarly, the cost of long-term care in Quebec was calculated at \$55,936 based on non-Quebec data using an average daily cost of long-term 
care of \$147.77 and a length of stay of 365 days (assuming a permanent stay). A relative daily cost of long-term care based on relative Case Mix Index was not available.

\section{Statistical analysis}

For each IPF individual, the annual health care resource utilization and cost relative to the index date was estimated as a pre-index year or as a post-index year. In addition, an incremental cost analysis was conducted using as a reference the second year prediagnosis costs. We used the second year before diagnosis as the baseline period to compare costs before and after the index IPF diagnosis date since the time between symptoms and diagnosis has been reported to be between 6 months and 2 years. ${ }^{9}$

To deal with censoring when calculating total costs postdiagnosis (ie, costs of patients who died not available), statistical methods ${ }^{10}$ were used to weight the uncensored costs with survival probability (or inverse probability weighting) to produce an unbiased estimate of the incremental costs associated with IPF. The steps to create the survival probability weighting are commonly known as the Lin method, ${ }^{10}$ and this includes four steps: 1) separating costs by annual year of follow-up pre- and postdiagnosis; 2) estimating average costs for each year for those patients who were alive at the beginning of the year; 3 ) multiplying the average annual costs by the Kaplan-Meier survival probability of being alive at the beginning of the interval; and 4) calculating the mean total costs by summing up these weighted costs over the complete study period. Consistent with the established literature, ${ }^{11}$ individual cost components (eg, cost of physician visits) were not adjusted for censoring because the different types of health care resource use that make up the total cost have a common pattern of censoring. Costs are presented in 2016 Canadian dollars.

\section{Results}

\section{Prevalence and incidence of IPF}

For the province of Quebec for the 5-year period, the number of cases who had an admission with an ICD-10-CA code of
J84.1 was 11,577 and the number of cases with an ICD-9-CM physician billing code of 516.3 was 372 cases (Table 1). Combined, this represented 11,820 unique cases in the data set with an IPF code. After excluding 1,241 cases that had a non-IPF interstitial lung disease code on a date which was after their last IPF code, there were 10,579 IPF cases based on a broad definition. The number of IPF cases based on a narrow definition was 8,683 . The prevalence rates for the board and narrow definition were 78.4 and 72.7 per 100,000 population.

Using our definition of incident cases (no IPF code in the 2 previous years), the annual incidence rate of IPF with a broad definition was $21.7 / 100,000$ population for women, 29.9/100,000 population for men, and 25.8/100,000 overall (Table 2). With a narrow definition, the annual incidence after adjusting for the 3 year capture was 18.4/100,000 population for women, $25.1 / 100,000$ population for men, and 21.7/100,000 overall. Table 2 also provides the detailed incidence rates by gender and age group for broad and narrow definitions.

\section{Cohorts description}

There were no statistical differences between the broad and narrow definition cohorts in terms of average age, percentage that are men, mean number of comorbidity, or proportion of the cohort with each type of comorbidities (Table 3). Overall, the average age of IPF at diagnosis was 77 years, and almost $60 \%$ of the cases were men. The average number of comorbid conditions was 4 , with the most common conditions being pulmonary disease $(81 \% ; 81 \%)$, pulmonary infection $(55 \%$; $56 \%$ ), and coronary artery disease without myocardial infarction $(54 \% ; 55 \%)$.

\section{Mortality}

Using the 2008 incident population, the survival rates for the IPF cohorts were not statistically different between the broad $(\mathrm{N}=2,011)$ and narrow definition $(\mathrm{N}=1,673)$, with a 3-year survival rate of $39.9 \%$ for broad and $37.4 \%$ for narrow definition (Figure 1). The mean survival time was 553 days (1.5 years)

Table I Data capture and IPF cohort selection

\begin{tabular}{llll}
\hline Cohort selection & $\begin{array}{l}\text { Identified from hospital } \\
\text { admissions }\end{array}$ & $\begin{array}{l}\text { Identified from } \\
\text { physician visits }\end{array}$ & $\begin{array}{l}\text { Final } \\
\text { cohort }\end{array}$ \\
\hline Patients with admission/visit with IPF only (unique patients) & $1 \mathrm{II}, 577$ & 372 & $\mathrm{II}, 820$ \\
$\quad$ Less patients with other ILD diagnosis & $(577)$ & $(0)$ & $(1,24 \mathrm{I})$ \\
IPF cases based on broad definition, excluding other ILD diagnosis & $1 \mathrm{I}, 000$ & 372 & 10,579 \\
$\quad$ Less patients with no diagnostic testing (eg, CT scan) & $(4,594)$ & $(0)$ & $(1,896)$ \\
IPF cases based on narrow definition, excluding other ILD diagnoses and & 6,406 & 372 & 8,683 \\
patients without diagnostic testing & & & \\
\hline
\end{tabular}

Abbreviations: IPF, idiopathic pulmonary fibrosis; ILD, interstitial lung disease; CT, computed tomography. 
Table 2 Incidence of IPF by age and sex for Quebec

\begin{tabular}{|c|c|c|c|c|c|c|}
\hline \multirow[t]{2}{*}{ Age group, years } & \multicolumn{2}{|c|}{ Women } & \multicolumn{2}{|l|}{ Men } & \multicolumn{2}{|c|}{ Overall } \\
\hline & $\mathbf{N}$ & $\begin{array}{l}\text { Annual ratel } \\
100,000 \text { pop }\end{array}$ & $\mathbf{N}$ & $\begin{array}{l}\text { Annual ratel } \\
100,000 \text { pop }\end{array}$ & $\mathbf{N}$ & $\begin{array}{l}\text { Annual rate } / \\
100,000 \text { pop }\end{array}$ \\
\hline \multicolumn{7}{|l|}{ Broad definition } \\
\hline$<20$ & 8 & 0.3 & 10 & 0.4 & 18 & 0.3 \\
\hline $20-29$ & 11 & 0.7 & 7 & 0.4 & 18 & 0.6 \\
\hline $30-39$ & 13 & 0.8 & 18 & I.I & 31 & 1.0 \\
\hline $40-49$ & 45 & 2.6 & 56 & 3.1 & 101 & 2.8 \\
\hline $50-59$ & 144 & 8.0 & 175 & 9.9 & 319 & 8.9 \\
\hline $60-69$ & 358 & 26.5 & 593 & 46.7 & 951 & 36.3 \\
\hline $70-79$ & 698 & 84.6 & 1,126 & 163.1 & $\mathrm{I}, 824$ & 120.4 \\
\hline $80-89$ & 985 & 194.4 & 1,259 & $4 \mid 4.3$ & 2,244 & 276.9 \\
\hline$\geq 90$ & 336 & 293.0 & 274 & 729.6 & 610 & 400.7 \\
\hline Total & 2,598 & 21.7 & 3,518 & 29.9 & 6,116 & 25.8 \\
\hline \multicolumn{7}{|l|}{ Narrow definition } \\
\hline$<20$ & 6 & 0.2 & 9 & 0.3 & 15 & 0.3 \\
\hline $20-29$ & 10 & 0.7 & 5 & 0.3 & 15 & 0.5 \\
\hline $30-39$ & II & 0.7 & 11 & 0.7 & 22 & 0.7 \\
\hline $40-49$ & 38 & 2.2 & 40 & 2.2 & 78 & 2.2 \\
\hline $50-59$ & 123 & 6.8 & 143 & 8.1 & 266 & 7.4 \\
\hline $60-69$ & 294 & 21.7 & 495 & 38.9 & 789 & 30.1 \\
\hline $70-79$ & 574 & 69.6 & 946 & 137.0 & I,520 & 100.3 \\
\hline $80-89$ & 842 & 166.2 & 1,063 & 349.8 & 1,905 & 235.0 \\
\hline$\geq 90$ & 301 & 262.5 & $24 I$ & 641.7 & 542 & 356.1 \\
\hline Total & 2,199 & 18.4 & 2,953 & 25.1 & 5,152 & 21.7 \\
\hline
\end{tabular}

Abbreviations: IPF, idiopathic pulmonary fibrosis; pop, population.

Table 3 Comorbidities of incident IPF cases in Quebec, 20072011

\begin{tabular}{lll}
\hline Cohort description & $\begin{array}{l}\text { Broad } \\
\text { definition } \\
\text { cases }\end{array}$ & $\begin{array}{l}\text { Narrow } \\
\text { definition } \\
\text { cases }\end{array}$ \\
\hline $\mathrm{N}$ & 6,116 & 5,152 \\
Age years, mean (SD) (min, max) & $76.6(12.1)$ & $76.8(12.0)$ \\
& $(0,106)$ & $(2,106)$ \\
Men (\%) & $58 \%$ & $57 \%$ \\
Number of comorbid conditions, & $4.0(1.9)$ & $4.0(1.9)$ \\
mean (SD) (min, max) & $(0,13)$ & $(0,12)$ \\
Pulmonary disease & $81 \%$ & $81 \%$ \\
Pulmonary infection & $55 \%$ & $56 \%$ \\
Coronary artery disease not myocardial & $54 \%$ & $55 \%$ \\
infarction & & \\
Heart failure & $35 \%$ & $36 \%$ \\
Diabetes & $35 \%$ & $35 \%$ \\
Atrial fibrillation & $25 \%$ & $25 \%$ \\
Fatigue & $20 \%$ & $20 \%$ \\
Lung cancer & $18 \%$ & $19 \%$ \\
Cardiovascular disease & $19 \%$ & $19 \%$ \\
Myocardial infarction & $10 \%$ & $11 \%$ \\
Depression & $11 \%$ & $11 \%$ \\
Pulmonary embolism & $9 \%$ & $9 \%$ \\
Sleep apnea & $9 \%$ & $8 \%$ \\
Gastroesophageal reflux disease & $8 \%$ & $8 \%$ \\
\hline Abbreviations: & $5 \mathrm{~F}, 1 \%$ \\
\hline
\end{tabular}

Abbreviations: IPF, idiopathic pulmonary fibrosis; SD, standard deviation. from the index diagnosis date for the broad cohort and 523 days (1.4 years) for the narrow cohort (test of homogeneity $p<0.001$, indicating no difference in survival curves).

\section{Health care resource utilization}

Table 4 presents, for the broad and narrow definitions, the mean annual health care resource utilization for the data available at each year before and after diagnosis. Of the 6,116 incident cases identified using the board definition and 5,152 using the narrow definition, more than $95 \%$ of cases had 3 years of data available prior to diagnosis; almost two-thirds had 4 years of data prior to diagnosis, while one third had 5 years of data prior diagnosis. Due to high mortality rates, the number of patients contributing to the analysis after diagnosis drops rapidly (eg, 39\% after 2 years postdiagnosis).

There was a consistent pattern in the use of health care resources for all care components as illustrated in Table 4. Overall, the average health care resource utilization per patient started increasing several years before diagnosis. In the first year after diagnosis, resource utilization continued to rise and then remained high for up to 2 years postdiagnosis for both the broad and narrow definition (Table 4). 


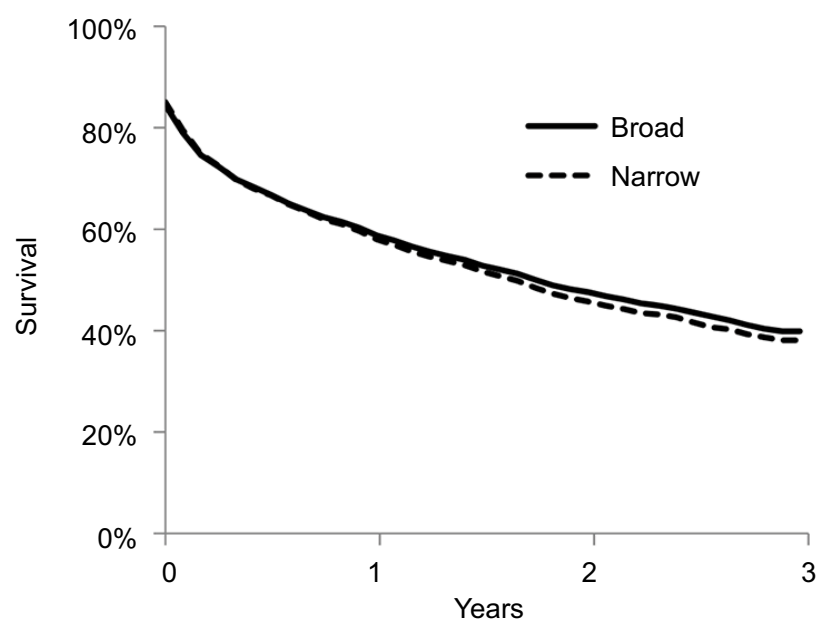

\begin{tabular}{|l|c|c|c|c|}
\hline \multirow{2}{*}{} & \multicolumn{4}{|c|}{ Follow-up } \\
\cline { 2 - 5 } & 0 & 1 year & 2 years & 3 years \\
\hline Broad definition, mean survival 553 days (95\% Cl: 516,550$)$ \\
\hline Number at risk & 2,011 & 1,212 & 968 & 37 \\
\hline Survival & $100.0 \%$ & $58.8 \%$ & $47.6 \%$ & $39.9 \%$ \\
\hline Narrow definition, mean survival 523 days (95\% Cl: 504,541$)$ \\
\hline Number at risk & 1,673 & 998 & 776 & 32 \\
\hline Survival & $100.0 \%$ & $61.1 \%$ & $46.8 \%$ & $37.4 \%$ \\
\hline
\end{tabular}

Figure I Kaplan-Meier survival estimates for incident IPF cases identified in 2008 with 3 year follow-up. Abbreviations: $\mathrm{Cl}$, confidence interval; IPF, idiopathic pulmonary fibrosis.

\section{Costs associated with IPF}

Consistent with the pattern observed for the consumption of health care resources, the costs associated with IPF increased before and after a diagnosis of IPF and stayed elevated up to 2-years following diagnosis (Table 5). More specifically, the average annual cost per patient 2 years prior to IPF diagnosis (broad definition) was $\$ 7,049$. In the year prior to diagnosis, the average annual cost per patient rose to $\$ 11,664$ (a $65 \%$ increase). The costs associated with the index diagnosis were $\$ 15,281$, and the average annual cost in the first year after diagnosis (not including index admission) was $\$ 15,281$. The average cost remained above $\$ 12,000$ for the second year postdiagnosis.

Table 5 also presents the incremental costs in reference to the annual cost observed in the second year prior to IPF diagnosis. Over the study period, the cumulative multiyear incremental cost relative to the 2 year prediagnosis baseline cost was $\$ 37,392$ (95\% confidence interval: $\$ 32,255$,
$\$ 42,541)$ for the broad definition. The proportion of incremental cost for the broad cohort was driven by admissions (59\% of total) and long-term care (25\%), while other cost components accounted for less change in overall incremental costs, home care ( $8 \%)$, specialists billings $(6 \%)$, general practice billings (3\%), and drugs (2\%). Similar incremental cost patterns were seen for the narrow definition cohort.

\section{Discussion}

This is the first study that tracks IPF patients over multiple years to document health care resource utilization and costs over time. Our results are important for many reasons. First, the results indicate that annual health care costs for patients with IPF begin to increase prior to diagnosis, which is consistent with the natural history of the disease. With the progression of the disease, a patient experiences declines in health status, which leads to investigation with high-resolution CT scan, lung biopsy, or bronchoscopy to help establish the 


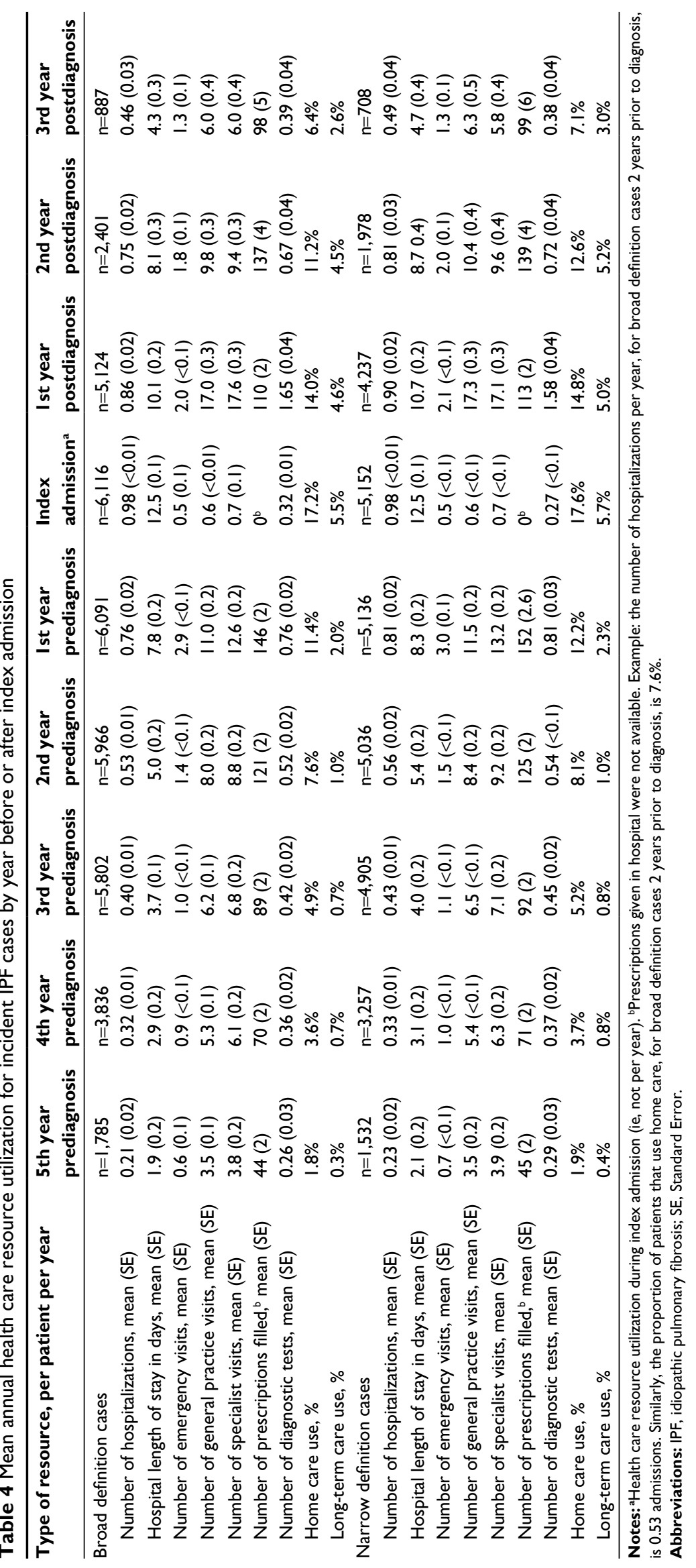




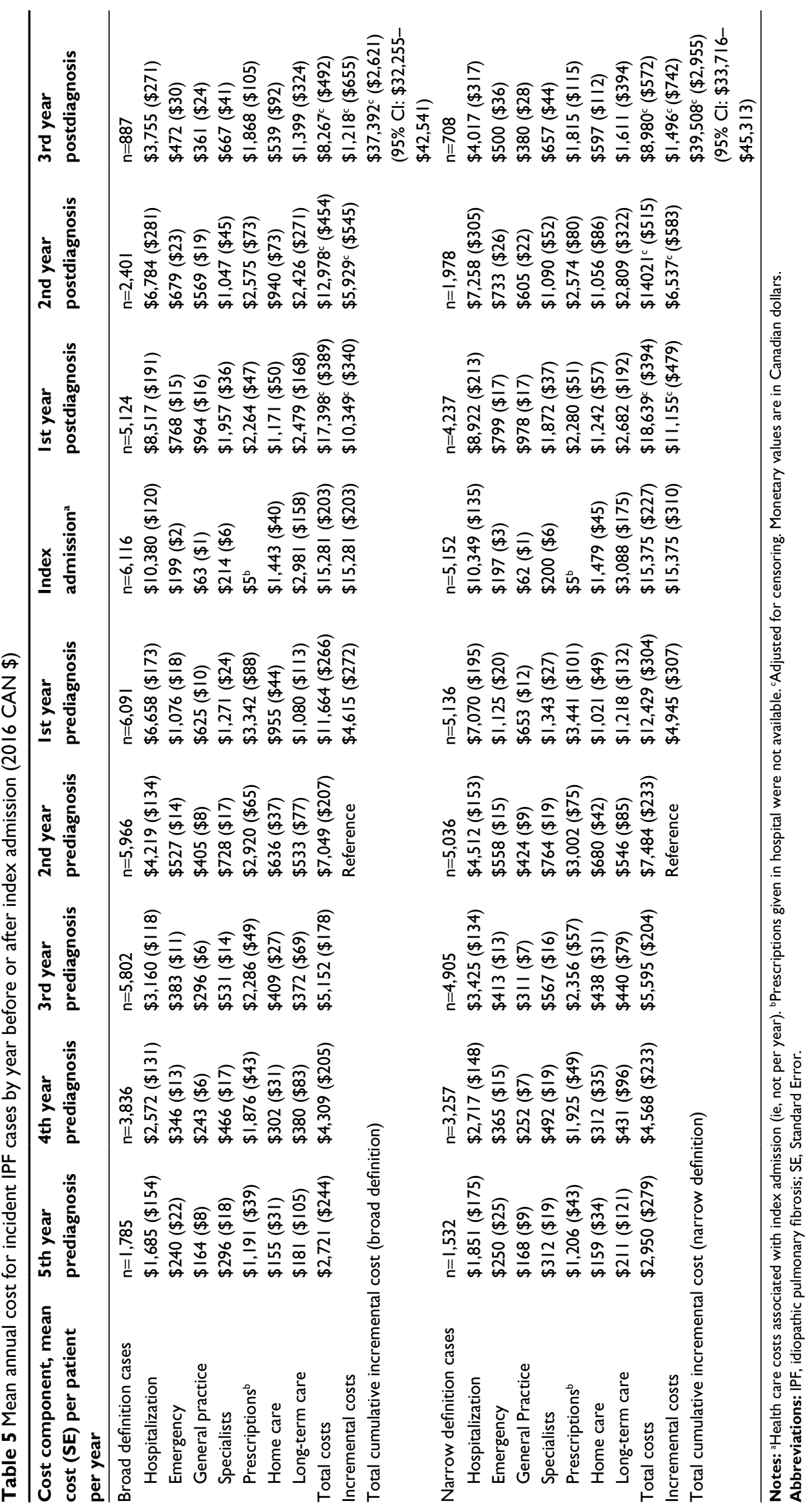


diagnosis. Our results indicate that the IPF costs per patient were relatively constant up to 2 years postdiagnosis.

The present estimate of the incidence of IPF for the province of Quebec expands on our previous work ${ }^{3}$ on Canadian estimates of IPF for the same period of time. Contrary to our previous study, we also had access to the physician database of Quebec, allowing us to identify IPF cases through physician billings in addition to hospital admissions. As a result, the incidence of IPF as defined by the broad and narrow case definitions is higher in the present study than those we previously reported, as we were able to capture outpatient data in addition to hospital-based data.

This study provides new information on the incremental health care resource utilization and costs associated with IPF. Contrary to previous studies, we provided up to 3 years of data following IPF diagnosis, and the results indicate that the health care use and costs remain higher than prediagnosis levels for the first 2 years following diagnosis. However, our 1-year results are somewhat comparable with a US study based on 7,855 IPF cases. ${ }^{12}$ In the Collard study, the average annual cost per patient rose from $\$ 10,124$ US dollars for the 1-year prediagnosis period to $\$ 20,887$ for the 1-year postdiagnosis period, due to increase utilization of hospitals, physician office visits, long-term care, and home care. In comparison, our average annual cost for the broad definition cohort rose from \$11,644 Canadian dollars the 1 -year prediagnosis to $\$ 17,398$ the 1 -year postdiagnosis, excluding the cost of index admission of $\$ 15,281$. Other studies in the US have documented an increase in health care resource utilization 1-year after IPF diagnosis; ${ }^{5,6,13}$ however, direct comparisons are difficult due to different health care systems. In addition, our data was prior to the approval of pirfenidone and nintedamib in Canada, which would impact the costs of managing IPF. Our study adds to this body of evidence by providing long-term cost data and showing that, compared to 2 years before IPF diagnosis, costs remain high even 2 years after diagnosis.

When compared to other Canadian burden of illness studies, the direct cost of IPF is higher than that of asthma, COPD, and lung cancer. For example, the average annual cost associated with medical care and wage loss due to asthma in Ontario was $\$ 1,280$ in 1998. ${ }^{14}$ Admissions accounted for $\$ 553$ per person, or $43 \%$ of costs. Even with severe asthma, the average per-person annual cost was $\$ 3,905 .{ }^{14}$ Similarly, the average per-person annual cost of medical care for COPD, based on a sample of COPD patients across Canada during the period 2009-2010, was $\$ 2,739$ for GOLD stage I patients, which rose to $\$ 6,241$ for patients with COPD GOLD stage
IV. ${ }^{15}$ For patients with severe COPD who have an exacerbation requiring an admission, the cost of the episode rose on average to $\$ 9,017$, which is lower than our cost estimates for IPF.

IPF may also have higher cumulative costs than lung cancer in Canada. Our results indicated that the average incremental cost of care for IPF in the first year after diagnosis was $\$ 25,630$ (including index admission). This is slightly lower than the first year cost of lung cancer in Ontario for the year 2007 which was estimated at $\$ 30,550 .{ }^{16}$ However for lung cancer, Alberta data indicated that $69 \%$ of the total cost of medical care for lung cancer occurs within the first 3 months of diagnosis, which includes chemotherapy. ${ }^{17}$ Meanwhile, our results have shown that the cost of medical care for IPF remains high for up to 2 years or until death. The largest component of the cost of medical care for lung cancer was admissions, which accounted for $\$ 12,833$ if the patient survived and $\$ 19,689$ for admissions if the patient died. ${ }^{16}$ The cost of first year admissions was higher for IPF patients than for lung cancer, especially for survivors, with costs of $\$ 18,897$ for patients with at least 1 year survival, and $\$ 20,412$ for those who died.

There are a number of strengths of this analysis, including using linked data for an established population with a comprehensive single-source public health care system in the province of Quebec. As such, we had access to all IPF hospitalizations and physician visits in Quebec. Quebec represents $23 \%$ of the population of Canada, and so our results should be representative of other jurisdictions across Canada or other publicly funded health care systems elsewhere. We were able to track patients for up to 5 years to identify their history and postdiagnosis costs. In addition, our estimates of the risk of mortality may be more robust because our health care databases were also linked to vital statistics data for the province which records the date of death for all causes at home or in the hospital (as opposed to hospital mortality only as in our previous work). In addition, we had access to a physician database, allowing us to identify additional cases of IPF.

Despite the strengths of this study, a number of limitations should be noted. First, to identify IPF patients, we relied on ICD-10-CA codes for admission, similar to our previous work, but also we used ICD-9-CM codes for physician billings. A recent study ${ }^{18}$ demonstrated that the use of ICD-9 codes falsely labels some interstitial lung disease patients as having IPF and misses some patients with IPF. However, our study uses a methodology for identifying IPF cases which is widely accepted and similar to other economic studies of IPF, allowing us to compare our results to other published 
reports, which would have the same errors in case identification. Unfortunately, the number of cases identified with ICD-9 codes alone was rare, since physicians typically record a general diagnosis (eg, pulmonary fibrosis) rather than a specific disease code (eg, idiopathic pulmonary fibrosis), and only the designated services performed by physicians are mandatorily recorded for reimbursement. This is an inherent limitation of data from administrative databases that are used for billing rather than research purposes; however, the physician billing database was very useful in establishing narrow definition cases since the diagnostic tests are mandatory fields. Narrow and broad definition cases appear to be similar in terms of comorbidities, mortality, health care utilization, and costs, and the presence of codes for diagnostic tests may have been previously underreported. In particular, the broad and narrow definition cohorts can be expected to be similar if, similar to Quebec, CT scanning is considered essential to establish IPF. There is also a limitation with the ICD coding for identification of cases, where ICD-10-CA J84.1 includes other diseases (diffuse pulmonary fibrosis, fibrosing alveolitis [cryptogenic], Hamman-Rich syndrome, and usual interstitial pneumonia). This study was undertaken prior to the reclassification of idiopathic interstitial pneumonias $^{19}$ and, while the other diseases might be rare, the use of J84.1 might have contributed to the presence of false-positive cases, especially for patients aged less than 50, given that IPF typically appears later in life.

Another important limitation is that we did not have access to cost data for the hospitalizations and ER visits and used Canadian non-Quebec data to derive these costs in Quebec based on our previous study. Although this is a limitation of our study, this assumption should have a minimal impact on our results as the populations of the current Quebec study and our previous study are similar ${ }^{3}$ in terms of age, gender, and length of stay. We also were not able to differentiate IPF-specific health care resource utilization or costs versus other non-IPF related care. We also had no information on nonmedical costs incurred by patients and their caregivers.

\section{Conclusion}

Within these limitations, our results suggest that previous studies that have focused on analyses 1-year pre- and postdiagnosis have underestimated the economic burden of IPF, which continues to be high even several years after the diagnosis date. Our results support the use of longitudinal data to study the burden of IPF over many years to take into account the clinical progression of IPF. To better inform policymak- ers, physicians, and patients, future areas of research include documenting HRQoL over several years following IPF diagnosis and evaluating indirect costs associated with IPF.

\section{Acknowledgments}

Provincial data were provided by Régie de l'Assurance Maladie du Québec (RAMQ). This research was funded by InterMune Canada Inc., which has since been acquired by F Hoffmann-La Roche Ltd. The sponsor had no role in the design and conduct of the study, in the analysis and interpretation of data, in the writing of the manuscript, or in the decision to submit the manuscript for publication.

\section{Disclosure}

JET, RBH, and NB declare grants from InterMune Canada Inc. (which has since been acquired by F. Hoffman-La-Roche) during the conduct of the study. CDF declares grants and personal fees from Roche Canada and Boehringer Ingelheim, and grants from Canadian Pulmonary Fibrosis Foundation, outside the submitted work. GD declares personal fees from Roche and Boehringer Ingelheim, outside the submitted work. MK declares grants and personal fees from Roche, Boehringer Ingelheim, GSK, Gilead, Prometic, and Alkermes; grants from Actelion, Respivert, and Synairgen; and personal fees from Astra Zeneca and Genoa, outside the submitted work. The authors report no other conflicts of interest in this work.

\section{References}

1. Raghu G, Collard HR, Egan JJ, et al. An official ATS/ERS/JRS/ALAT statement: idiopathic pulmonary fibrosis: evidence-based guidelines for diagnosis and management. Am J Respir Crit Care Med. 2011;183(6):788-824.

2. Lee AS, Mira-Avendano I, Ryu JH, Daniels CE. The burden of idiopathic pulmonary fibrosis: an unmet public health need. Respir Med. 2014;108(7):955-967.

3. Hopkins RB, Burke N, Fell C, Dion G, Kolb M. Epidemiology and survival of idiopathic pulmonary fibrosis from national data in Canada. Eur Respir J. 2016;48(1):187-195.

4. Fell CD. Idiopathic pulmonary fibrosis: phenotypes and comorbidities. Clin Chest Medicine. 2012;33(1):51-57.

5. Collard HR, Ward AJ, Lanes S, Cortney HD, Rosenberg DM, Hunsche E. Burden of illness in idiopathic pulmonary fibrosis. $J$ Med Econ. 2012;15(5):1-7.

6. Wu N, Yu YF, Chuang CC, Wang R, Benjamin NN, Coultas DB. Healthcare resource utilization among patients diagnosed with idiopathic pulmonary fibrosis in the United States. J Med Econ. 2015;18(4): 249-257.

7. Canadian Institute for Health Information. Canadian MIS DatabaseHospital Financial Performance Indicators, 1999-2000 to 2009-2010, Methodological Notes. Ottawa: CIHI; 2011.

8. Canadian Institute for Health Information. Your Health System: Cost of a Standard Hospital Stay. Available from: http://yourhealthsystem.cihi. ca/hsp/inbrief?lang=en\#!/indicators/015/cost-of-a-standard-hospitalstay-cshs/;mapC1;mapLevel2;trend(C1);/. Accessed August 4, 2017. 
9. Kim DS, Collard HR, King TE Jr. Classification and natural history of the idiopathic interstitial pneumonias. Proc Am Thorac Soc. 2006;3(4):285-292.

10. Lin DY, Feuer EJ, Etzioni R, Wax Y. Estimating medical costs from incomplete follow-up data. Biometrics. 1997;53(2):419-434.

11. Ye $X$, Henk HJ. An introduction to recently developed methods for analyzing censored cost data. ISPOR Connections. 2007;13(4):11-13.

12. Collard HR, Chen SY, Yeh WS, et al. Health care utilization and costs of idiopathic pulmonary fibrosis in U.S. Medicare beneficiaries aged 65 years and older. Ann Am Thorac Soc. 2015;12(7):981-987.

13. Raimundo K, Chang E, Broder MS, Alexander K, Zazzali J, Swigris JJ. Clinical and economic burden of idiopathic pulmonary fibrosis: a retrospective cohort study. BMC Pulm Med. 2016;16:2.

14. Ungar WJ, Coyte PC, Chapman KR, MacKeigan L. The patient level cost of asthma in adults in south central Ontario. Pharmacy Medication Monitoring Program Advisory Board. Can Respir J. 1998;5(6): $463-471$
15. Maleki-Yazdi MR, Kelly SM, Lam SY, Marin M, Barbeau M, Walker V. The burden of illness in patients with moderate to severe chronic obstructive pulmonary disease in Canada. Can Respir J. 2012;19(5):319-324.

16. de Oliveira C, Bremner KE, Pataky R, et al. Understanding the costs of cancer care before and after diagnosis for the 21 most common cancers in Ontario: a population-based descriptive study. CMAJ Open. 2013;1(1):E1-E8.

17. Demeter SJ, Jacobs P, Chmielowiec C, et al. The cost of lung cancer in Alberta. Can Respir J. 2007;14(2):81-86.

18. Ley B, Urbania T, Husson G, et al. Code-based diagnostic algorithms for idiopathic pulmonary fibrosis. Case validation and improvement. Ann Am Thorac Soc. 2017;14(6):880-887.

19. Travis WD, Costabel U, Hansell DM, et al. An official American Thoracic Society/European Respiratory Society statement: Update of the international multidisciplinary classification of the idiopathic interstitial pneumonias. Am J Respir Crit Care Med. 2013;188(6):733-748.
ClinicoEconomics and Outcomes Research

\section{Publish your work in this journal}

ClinicoEconomics and Outcomes Research is an international, peerreviewed open-access journal focusing on health technology assessment, pharmacoeconomics and outcomes research in the areas of diagnosis, medical devices, and clinical, surgical and pharmacological intervention. The economic impact of health policy and health systems
Submit your manuscript here: https://www.dovepress.com/clinicoeconomics-and-outcomes-research-jout

organization also constitute important areas of coverage. The manuscript management system is completely online and includes a very quick and fair peer-review system, which is all easy to use. Visit http://www.dovepress.com/testimonials.php to read real quotes from published authors. 